\section{Verzögertes Erbrechen nach Cisplatin}

\begin{abstract}
In einer randomisierten Studie wurde Metoclopramid/Dexamethason mit Aprepitant/Dexamethason bei Patienten verglichen, die Aprepitant/Palonosetron/Dexamethason zur Prophylaxe akuten Erbrechens erhalten hatten.
\end{abstract}

Z ur Prophylaxe des akuten Erbrechens (binnen 24 Stunden nach Therapiebeginn) bei Cisplatin-basierter Chemotherapie empfehlen aktuelle Leitlinien die Kombination aus dem NK1-Rezeptor-Antagonisten Aprepitant, einem 5-HT ${ }_{3}$-Rezeptor-Antagonisten und Dexamethason. Für die Zeit danach, also das verzögerte Erbrechen, ist die Studienlage bislang uneinheitlich. Deshalb wurde der bisherige Standard Aprepitant/Dexamethason mit Metoclopramid/Dexamethason verglichen.

Krebspatienten, die vor Beginn der Cisplatin-basierten Chemotherapie 0,25 mg Palonosetron i.v., 12 mg Dexamethason und $125 \mathrm{mg}$ Aprepitant oral erhalten hatten, wurden an den Tagen
2-4 auf entweder orales Dexamethason $8 \mathrm{mg}$ plus Aprepitant $80 \mathrm{mg}$ 1-mal täglich (A + D, Tag 2 und 3; $n=147)$ oder Metoclopramid $20 \mathrm{mg}$ 4-mal täglich plus Dexamethason $8 \mathrm{mg}$ 2-mal täglich $(\mathrm{M}+\mathrm{D}, \mathrm{n}=137)$ randomisiert. Primärer Endpunkt war das komplette Ansprechen, d.h. kein Erbrechen und keine zusätzliche Therapie an Tag 2-5.

Die Prophylaxe des akuten Erbrechens gelang in beiden Gruppen gleichermaßen. Doch auch an den Tagen 2-5 gab es bei der Rate für das komplette Ansprechen keine signifikanten Unterschiede: $80,3 \% A+D$ versus $82,5 \% M+D$ ( $\mathrm{p}<0,38$ ). Die sekundären Endpunkte (vollständige Kontrolle, keine Übelkeit etc.) fielen ebenfalls gleich aus ( $p<0,24)$.
Nicht ausgeschlossen werden kann, dass die gute Effizienz beider Kombinationen auf die lange Wirksamkeit des 5- $\mathrm{HT}_{3}$-Rezeptor-Antagonisten Palonosetron zurückzuführen ist. Ob sich ähnliche Ergebnisse auch mit kürzer wirksamem Ondansetron oder Granisetron erreichen lassen, bleibt zu überprüfen.

Fazit: Bei Krebspatienten, die dieselbe Therapie zur Prophylaxe des akuten Erbrechens bei Cisplatin-basierter Chemotherapie erhalten, ist in der Prophylaxe des verzögerten Erbrechens Aprepitant/ Dexamethason der Kombination Metoclopramid/Dexamethason nicht überlegen. Nach Ansicht der Forscher sollte dies unter dem Kostenaspekt zu einem Umdenken in den aktuellen Empfehlungen führen.

Kathrin von Kieseritzky

Roila F et al. Aprepitant versus metoclopramide, both combined with dexamethasone, for the prevention of cisplatin-induced delayed emesis: a randomized, double-blind study. Ann Oncol. 2015;26(6):1248-53.

\title{
Zielgerichtete Therapien: Xerosis bisher unterbewertet
}

\section{Molekular gezielte Therapien haben im Vergleich zur Chemotherapie ein günstigeres Nebenwirkungsprofil. Doch schädigen einige der Substanzen die Haut. Dermatologen untersuchten die Inzidenz der wenig beachteten Xerosis.}

$\mathrm{D}$ ermatologische Nebenwirkungen unter gezielten Therapien, z. B. mit Tyrosinkinasehemmern (TKI), sind zwar meist nicht lebensbedrohlich, beeinträchtigen den Patienten aber subjektiv stark und können sogar zum Ab-

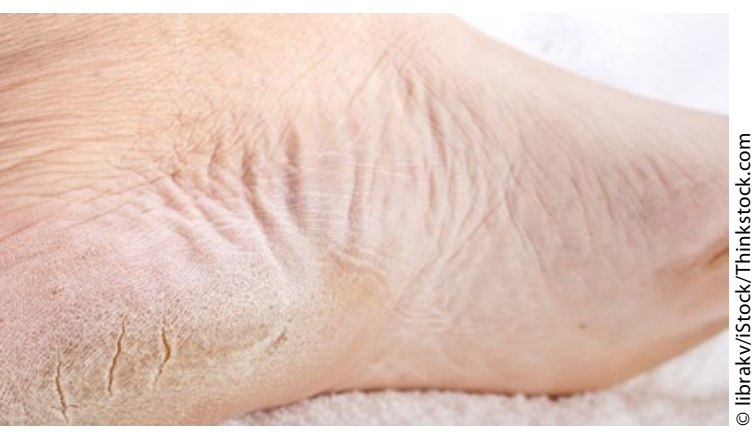

Xerosis, hier am Fuß, wird als Nebenwirkung zielgerichteter Therapien häufig unterschätzt. bruch der lebensverlängernden Behandlungen führen. Unter den dermatologischen Toxizitäten wurde der als harmlos geltenden Xerosis bisher wenig Aufmerksamkeit zuteil. Onkologen schätzen möglicherweise ihre Inzidenz geringer ein als Dermatologen, auch wird die psychische Belastung der Patienten, z. B. durch Juckreiz, leicht übersehen. In einer Metaanalyse sollte die Inzidenz der Xerosis unter zielgerichteten Therapien evaluiert werden.

130 Publikationen zu Phase-II- oder -III-Studien mit insgesamt 58 gezielten Therapien gingen in die Analyse ein. Die Xerosis-Inzidenz betrug insgesamt $17,9 \%$. Eine schwere Xerosis trat insgesamt bei 1,0\% der Patienten auf. Das relative Risiko, im Vergleich zu Placebo eine Xerosis jedweden Grades zu entwickeln, betrug in einer rechnerischen $\mathrm{Zu}$ sammenschau von 16 randomisierten und kontrollierten Studien - überwiegend mit TKI $-2,99$ und zeigte deutliche Unterschiede zwischen den Substanzen $(\mathrm{p}<0,001)$. Das Risiko war unter Sorafenib am niedrigsten und unter Gefitinib am höchsten. Allerdings könnte die Dokumentation der Xerosis in den einzelnen Zentren unterschiedlich gehandhabt worden sein, auch dürften Komorbiditäten oder Begleitmedikation die Xerosis-Häufigkeit beeinflusst haben.

Fazit: Patienten unter zielgerichteten Therapien haben ein nicht unerhebliches Risiko für eine Xerosis. Meist ist diese Nebenwirkung mild, doch kann in schwereren Fällen die Lebensqualität deutlich gemindert sein. Die Patienten sollten frühzeitig an den Dermatologen überwiesen werden, um die Behandlung zielführend und ohne Dosisreduktion fortsetzen zu können. Barbara Kreutzkamp

Valentine J et al. Incidence and risk of xerosis with targeted anticancer therapies. J Am Acad Dermatol. 2015;72(4):656-67. 